\title{
TREATMENT OF DEEP CORNEAL ULCERS IN DOGS WITH PORCINE SMALL INTESTINAL SUBMUSOSA USING A MODIFIED SURGICAL TECHNIQUE
}

\author{
Andrea Steinmetz ${ }^{1}$ and Lars Theyse ${ }^{1}$ \\ ${ }^{1}$ Universitat Leipzig Veterinarmedizinische Fakultat
}

September 24, 2020

\begin{abstract}
Deep corneal ulcers of four brachycephalic dogs were treated by a modified surgical technique using small intestinal submucosa (SIS). SIS was cut to exact size of the defect but not sutured. Afterwards a temporary nictitating membrane flap was placed. The outcome was very good in all cases.
\end{abstract}

\section{Key clinical message}

Using a piece of SIS trimmed to fill the corneal ulcer without suturing in combination with a third eyelid flap may be useful in selected cases (e.g. short procedure time is imperative, surgeon experience is lacking, financial restrictions).

Key words: dog, BOS, SIS, corneal ulcer, modified technique, nictitating membrane flap

\section{Introduction}

In cases of deep corneo-stromal ulceration surgical therapy is generally indicated. To improve healing, replace lost tissue and to achieve best possible tectonic and visual outcome autogenic, allogenic and xenogenic grafts and biomaterials have been used. Xenogenic grafts and biomaterials can be considered if autogenous or allogenous corneal grafting is not possible because of the extent of the defect or failing availability. Porcine bladder acellular matrix (ACell), amniotic membranes, bovine pericardium and swine intestinal submucosa (SIS) are biomaterials which have been applied already in treating canine corneal defects. Typically, these materials are anchored at the edge of the defect to the intact peripheral corneal tissue with sutures. The aim of this study was to demonstrate a modified technique not requiring corneal suturing and in that way making the procedure easier to perform.

\section{Case history and examination}

Four middle aged brachycephalic dogs (three French Bulldogs, one Pug) were included in the study. The data breed, gender, age and eye are listed in table 1. All dogs were examined in general clinical and by slit-lamp-biomicroscopy (SL 17, Kowa company Ltd, Tokyo/Japan).

The general examination of all dogs in this case report revealed extreme loud breathing and stridor. All owners stated that their dog was very exercise and heat intolerant. These were signs of a severe respiratory brachycephalic syndrome.

The slit lamp examination showed typical signs of brachycephalic ocular syndrome (BOS) in both eyes and a deep stromal ulcer in one eye (fig. 3). Characteristics and causes of the ulcer, detected bacteria, their 
sensitivity to antibiotics, duration of third eyelid flap application, healing time, vision and transparency of the cornea at time during the last examination are listed in table 1 too.

Corneal bacteriological swab samples were taken in three cases. Bacterial swabs were positive with a monoculture of Streptococcus canis in case 1 and with Streptococcus canis and Enterobacter cloacae in case 3. From the corneal swab sample in one deep melting ulcer (case 4) no bacteria could be isolated.

\section{Treatment}

Local medical therapy was started with Moxifloxacin eye drops (Vigamox@), Alcon Pharma GmbH, Freiburg, Germany) six times in day, Atropine eye drops (Atropin-POS 1\%@, Ursapharm GmbH, Saarbrücken, Germany) s.i.d. and autologous serum eye drops five times per day. Systemic Cefalexin (Cefaseptinß), Vetoquinol, Ismaning, Germany) $20 \mathrm{mg} / \mathrm{kg}$ b.i.d. and $2 \mathrm{mg} / \mathrm{kg}$ Robenacoxib (Onsiorß, Elanco, Homburg, Germany) s.i.d were administered orally.

Surgery was performed within a range of three hours to two weeks after initial diagnosis. Anaesthesia was induced intravenously with $0.5 \mathrm{mg} / \mathrm{kg}$ diazepam (Faustanß), Temmler Pharma, Marburg, Germany) and $0.5 \mathrm{mg} / \mathrm{kg}$ levomethadone in a fixed combination with $0.025 \mathrm{mg} / \mathrm{kg}$ fenipramide (L-Polamivetß, Intervet, Unterschleißheim, Germany). Anaesthesia was maintained via endotracheal intubation with $1 \%$ isoflurane (Isofluran CP, CP Pharma, Burgdorf, Germany) dissolved in oxygen at a flow rate of $10 \mathrm{ml} / \mathrm{kg} / \mathrm{min}$.

After placing the dog in dorsal recumbency the head was stabilized in a vacuum-bag. The eye was prepared for surgery and a Barraquer eyelid speculum was inserted. A $15 \mathrm{~mm}$ SIS disc (Vetrix@ BioSIS ECM, Vetrix, Cumming, USA) was rehydrated in balanced salt solution (BSS@, Serag Wiessner, Naila, Germany) and trimmed to exact size of the ulcer thus filling the defect completely (fig. 1). The ulcer bed was not prepared before the SIS was placed. A nictitating membrane flap was placed by horizontal matress suture pattern (three stitches) in the upper eyelid with Nylon-suture 4-0 (Ethilonß, Ethicon, Flensburg, Germany). Care was taken not to dislodge the disc out of the defect during this procedure (fig. 2). Preoperative medication was continued with Moxifloxacin and Atropine applied directly on the nictitating membrane. Cefalexin and Robenacoxib were prescribed for two weeks and for four days, respectively.

The flap was left in place for a minimum of three weeks. The owners were instructed to look for purulent discharge and signs of pain during this period. The patients were re-examined at time of releasing the nictitating membrane flap (all cases), five days after releasing the nictitating membrane flap (one case) and three weeks after release (two cases).

\section{Outcome}

All dogs were comfortable during the three weeks with the third eyelid flap. No dog showed signs of pain and purulent discharge.

At the time of releasing the third eyelid flap, defects were completely covered with epithelium and all defects showed neovascularization. (fig 4) Cases 1 and 2 were lost for further follow up. Regression of the vascularization and increase of corneal transparency were clearly noted six weeks postoperatively in cases 3 and 4 (fig. 5). At time of final examination, the vision was excellent in all cases.

\section{Discussion}

The surgical indication was given in all four ulcers of this retrospective study because of the depth of the corneal defect (4/4), signs of melting (2/4), big diameter of the ulcus $(4 / 4)$ and poor healing conditions because of BOS (4/4).

Most commonly isolated bacteria in infected corneal ulcers are Staphylococcus and Streptococcus spp. In this study Streptococcus canis could be detected in two cases. In contrast, the corneal swab sample of the melting ulcer in case 4 was negative.

While waiting for the antibiotic test results all ulcers in this study were treated with systemic antibiotics and topical application of preservative free antibiotic eyedrops before and after surgery. 
Ulcerative keratitis has a vast range of causes in dogs. Brachycephalic breeds have the highest prevalence of corneal ulcers with a twenty times higher likelihood to be affected than non-brachycephalic dogs. The predisposing factors include macroblepharon, shallow orbits with subsequent lagophthalmos, medial canthal entropion, trichiasis, tear film deficits and reduced corneal sensitivity. These factors are collectively known as brachycephalic ocular syndrome (BOS). In one study most of the mean values of ocular surface diagnostic tests were lower in brachycephalic compared to non-brachycephalic dogs.

This study consisted of cases in which a therapeutic compromise had to be found. Therapeutic management was limited due to financial restraints (cases 2 and 4), compliance of the owners (cases 1 and 2) and anaesthetic restraints due to the severe respiratory brachycephalic syndrome (all cases). Surgical therapy was imperative in all four cases because of the deep of the ulcers. The compromise was to use a xenogenic biomaterial as scaffold and for replacing missing tissue without suturing in combination with a third eyelid flap for providing bandage and mechanical stability. These biomaterials have been used successfully for surgical therapy of corneal defects in dogs and cats and replace missing tissue und and serve for regeneration. They were applied either alone or in combination with covering by a conjunctival flap, with temporary lateral tarsorrhaphy and a temporary nictitating membrane flap. All these grafts were placed in the defect and sutured in the peripheral intact cornea tissue. This takes time and is an expensive surgery. In some instances a short procedure time is imperative and/or financial restrictions exist. Furthermore, suturing the cornea requires microsurgical equipment and experience of the surgeon. Sometimes one or both of these requirements are not met.

The main disadvantage of the third eyelid flap is to monitor progress or worsening.

However the third eyelid flap is superior to a temporary lateral tarsorrhaphy for full coverage of the nonfixated piece of SIS. In addition, in case of worsening pain and increasingly discharge would be expected and couldn't be seen in all four cases.

Further limitations of the study were the small sample size and the short follow up time in two cases. Studies with more patients and a comparison group for further research into this technique should be performed in future. But the first results presented in this case report are promising that the modified surgical technique may be useful in very selected cases because it makes the procedure easier and faster. In all cases of this study the cosmetic and visual outcome was very good to excellent.

\section{Acknowledgements and funding information}

We acknowledge support from the German Research Foundation (DFG) and funding of the Leipzig University within the program of Open Access Publishing.

\section{Author contributions:}

Author 1: surgeon, main author, writing of the most parts, composing the paper, figures

Author 2: composing the paper, writing parts of the discussion

\section{Conflict of interest}

There is no conflict of interest.

\section{Figure list}

Fig. 1 Exact trimmed SIS disc fills in the ulcer completely.

Fig. 2 Application of the nictitating membrane flap.

Fig. 3 Deep stromal large ulcers.

Fig. 4 Healing status a after removing of the third eyelid flap.

Fig. 5 Very good corneal transparency six weeks postoperatively. 

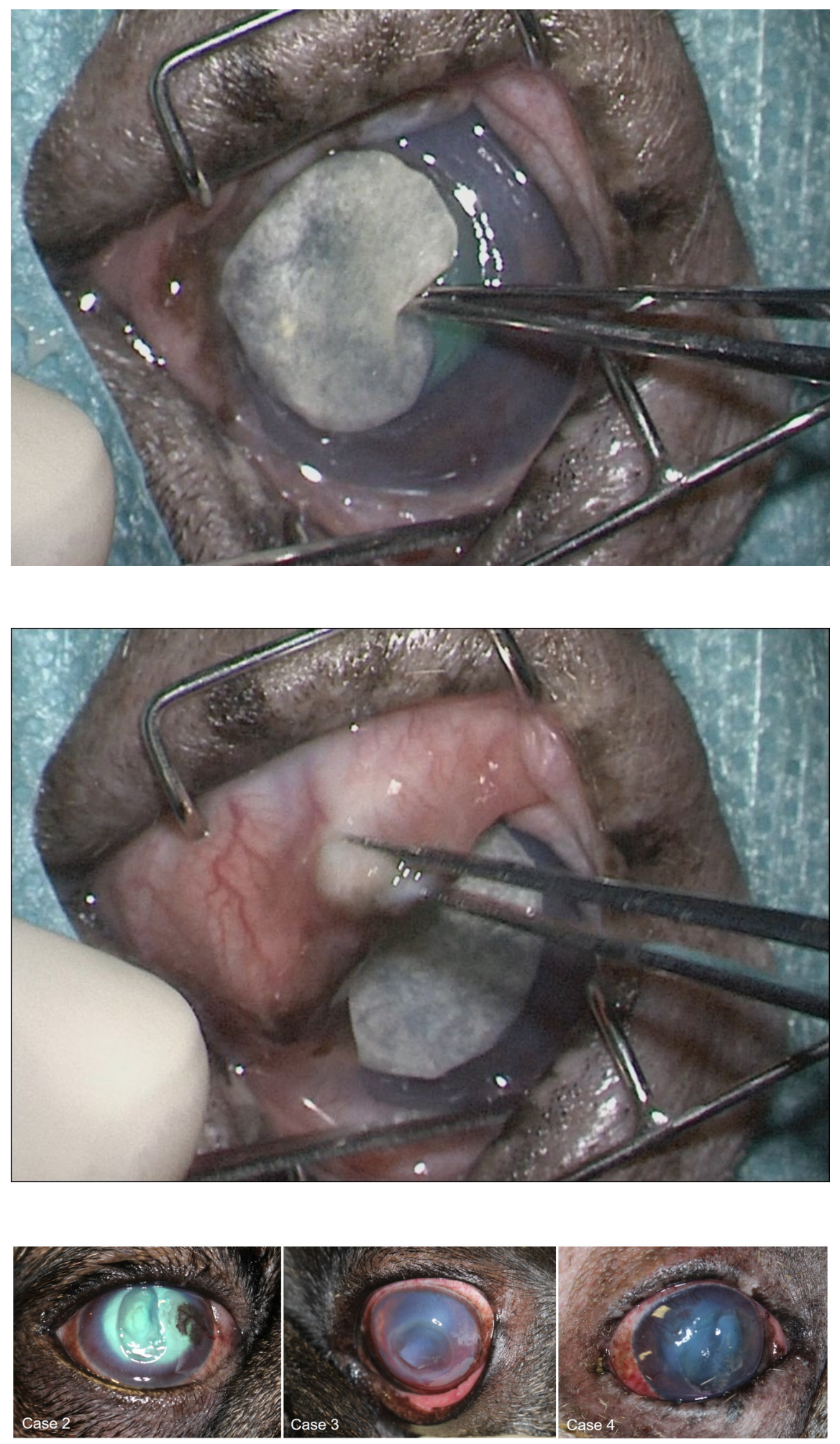

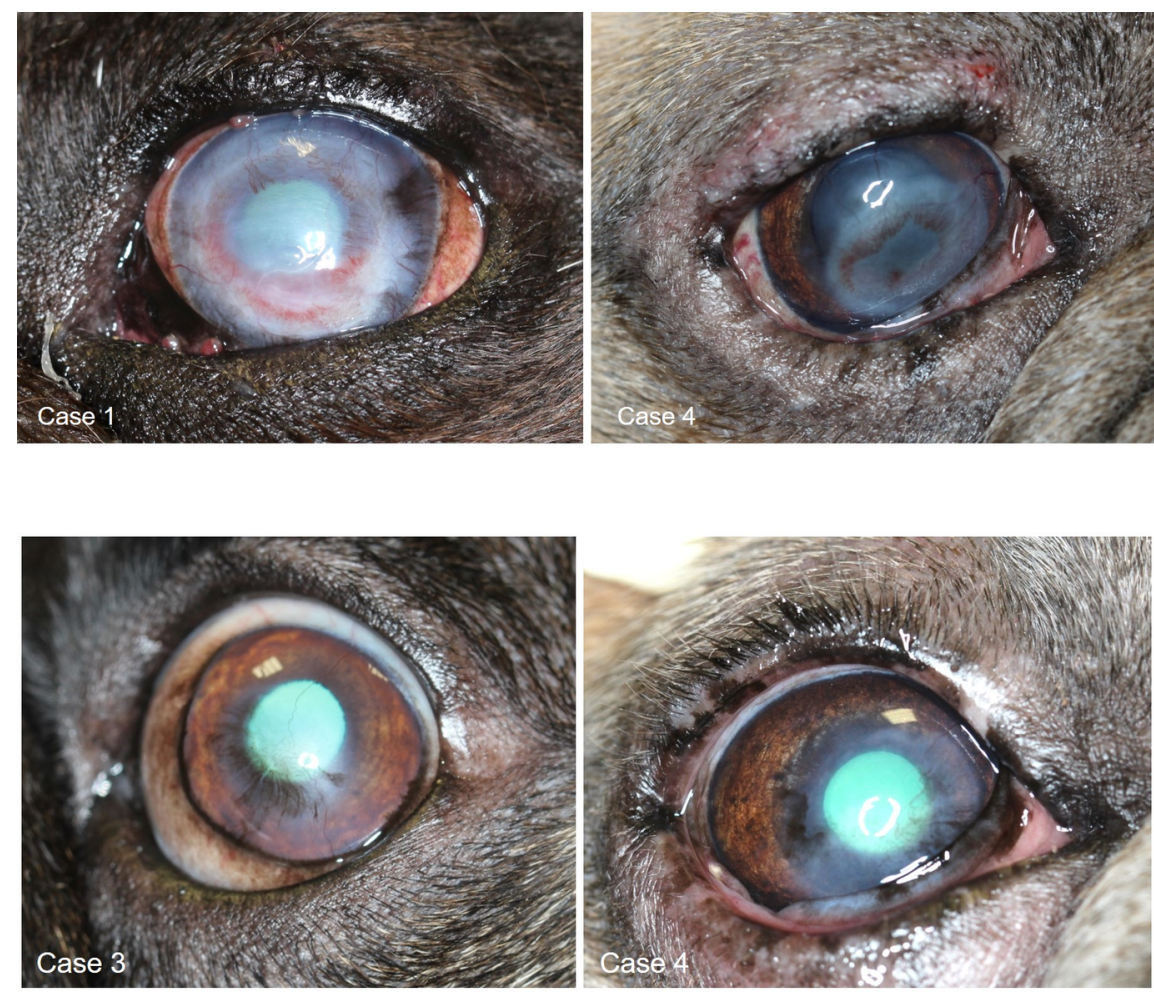